\title{
The melting curve of iron from quantum mechanics calculations
}

\author{
D. Alfè ${ }^{\mathrm{a}, \mathrm{b}, *}$, G.D. Price ${ }^{\mathrm{a}}$, M.J. Gillan ${ }^{\mathrm{b}}$ \\ ${ }^{a}$ Department of Earth Sciences, University College London, Gower Street, London WC1E 6BT, UK \\ ${ }^{\mathrm{b}}$ Department of Physics and Astronomy, University College London, Gower Street, London WC1E 6BT, UK
}

Received 20 May 2003; revised 20 November 2003; accepted 4 December 2003

\begin{abstract}
The high-pressure melting curve of iron is of major importance to the Earth's sciences, as it provides a close estimate of the temperature of the Earth's core. Despite being studied experimentally for more than a decade and, more recently, using theoretical quantum mechanics techniques, there are still large discrepancies between different groups. In this article, we will describe our theoretical approach to the problem and discuss the reason of the discrepancies with other theoretical calculations.

(C) 2004 Elsevier Ltd. All rights reserved.
\end{abstract}

\section{Introduction}

The thermodynamic properties of iron, and in particular its high-pressure melting curve, have been the subject of intense investigation in the past few decades. The reason for this substantial interest is that iron is most certainly the main constituent of the Earth's core, therefore, by knowing its melting temperature at the pressure of the inner-outer core boundary (ICB) one has a good estimate of the temperature of the core. However, the conditions of the ICB are so extreme that they are still out of reach of conventional static high-pressure experiments performed in diamond anvil cells (DAC). These methods allow investigation up to pressures and temperatures of about $200 \mathrm{GPa}$ and $3000 \mathrm{~K}$. Unfortunately though, despite the strenuous effort of the past few decades, the DAC experimental situation is still not resolved, with discrepancies between different groups of up to $500 \mathrm{~K}$ even at pressures as low as $60 \mathrm{GPa}$ [1-3]. To investigate the high-pressure region of the phase diagram one has to resort to shock wave techniques. In these experiments, one directly obtains the typical pressure versus volume relation called the Hugoniot, and in particular the $p, V$ point where melting occurs. More precisely, the two points $p_{i}, V_{i}$ and $p_{f}, V_{f}$ where melting begins and ends, respectively, with a two phase region in between. The temperature is not directly

\footnotetext{
* Corresponding author. Address: Department of Earth Sciences, University College London, Gower Street, London WC1E 6BT, UK.

E-mail address: d.alfe@ucl.ac.uk (D. Alfè).
}

accessible, and it is usually estimated by making assumptions on the specific heat and the Grüneisen parameter. A number of experimental data is available to date, and they fix the melting of iron on the Hugoniot in the pressure region 221-243 $\mathrm{GPa}$, with temperatures estimated in the range $5000-6000 \mathrm{~K}$.

Recent advances in computer power have made it possible for theoretical techniques, based on quantum mechanics methods, to be used to calculate the highpressure melting curve of iron from first principles. Two different approaches have been used in the past few years. The first is based on the calculation of free energies, with the melting temperature $T_{\mathrm{m}}$ defined at each fixed pressure by $G_{1}\left(p, T_{\mathrm{m}}\right)=G_{\mathrm{s}}\left(p, T_{\mathrm{m}}\right)$, where $G_{1}(p, T)$ and $G_{\mathrm{s}}(p, T)$ are the Gibbs free energies of liquid and solid, respectively; we call this the free energy approach [4-6]. The second is based on the direct simulations of solid and liquid in coexistence; we call this the coexistence approach $[7,8]$. The results obtained from different groups differ by as much as $1000 \mathrm{~K}$. The reasons for these discrepancies have been addressed in our previous paper [9], and can be summarised as follows. The coexistence simulations performed so far have been implemented using classical empirical potentials fitted to first principles calculations. Of course, in doing so one obtains the melting temperature of the potential employed, which is by no means guaranteed to be equal to the one which would be obtained directly using first principles simulations. We showed in our previous paper [9] that if the classical 
potential is close enough to the quantum mechanics system (in a sense which will become clear later in the article), one can develop a method to correct for the possible difference between the two melting temperatures, and we showed this by applying the corrections to a highpressure melting point of Fe calculated using the potential due to Belonoshko et al. [8]. This potential has also been used to study the stability of body centred cubic iron under Earth's core conditions [10], an issue that has attracted renewed interest recently [11].

Recently, thanks to the currently available computer power, it has even become possible to perform coexistence simulations directly from first principles, and we have calculated points on the low-pressure melting curve of aluminium to demonstrate this [12]. The excellent agreement obtained between the coexistence method [12] and the free energy approach [13] is an additional confirmation that, when applied consistently, the two techniques work equally well.

In this article, we will summarise the main ideas of the free energy approach and the coexistence approach and in addition to our previous calculations we also report our results from the coexistence approach for a low-pressure melting point of iron.

The article is organised as follows. In Section 2 we summarise the main ingredients used in our first principles calculations. In Section 3 we introduce the free energy approach, and we describe the techniques used to calculate the free energies of liquid and solid iron in Sections 3.1 and 3.2 , respectively. Section 3.3 contains the resulting melting curve with a discussion of errors. In Section 4 we discuss the coexistence method and the techniques employed to correct the melting temperature obtained from a classical potential, and in Section 4.1 we report the resulting melting temperatures. Section 5 contains discussion and conclusions.

\section{Technical details}

The technical details employed in our calculations on high-pressure $\mathrm{Fe}$ have been reported extensively in our previous papers $[5,6,14]$, so we give only a brief summary here. They are based on density functional theory (DFT) techniques [15]. The exchange-correlation functional $E_{\mathrm{xc}}$ is the generalised gradient approximation known as Perdew-Wang 1991 [16,17]. We use the projector-augmented-wave (PAW) implementation of DFT [14,18,19], a technique that shares the properties both of all-electron methods such as full-potential linearised augmented plane waves (FLAPW) [20] and the ultrasoft pseudopotential method [21]. The calculations were done using the VASP code [22,23], with the implementation of an efficient extrapolation of the electronic charge density [24]. Details of the core radii, augmentation-charge cut-offs, etc. are exactly as in our earlier papers $[5,6,14]$. Our division into valence and core states is also the same as before: the $3 p$ electrons are treated as core states, but their response to the high compression is represented by an effective pair potential, with the latter constructed using PAW calculations in which the $3 p$ states are explicitly included as valence states. Brillouin-zone (BZ) sampling was performed using Monkhorst-Pack (MP) special points [25]. The plane wave cut-off was $300 \mathrm{eV}$ in all calculations. The timestep used in the dynamical simulations was $1 \mathrm{fs}$.

The accuracy of DFT depends very much on the approximation used for the electronic exchange-correlation energy $E_{\mathrm{xc}}$. It is known that the local density approximation is not fully satisfactory for $\mathrm{Fe}$ [26], but that modern generalised-gradient approximations (GGA) reproduce a wide range of properties very accurately. These include the equilibrium lattice parameter, bulk modulus and magnetic moment of body-centred cubic (b.c.c.) Fe at ambient pressures [27-29], and the phonon dispersion relations of the b.c.c. phase [14,30]. There has been much DFT work on different crystal structures of $\mathrm{Fe}$ at high pressures, and experimental low-temperature results for the pressure as a function of volume $p(V)$ up to $p=300 \mathrm{GPa}$ for the h.c.p. structure are accurately predicted [31], although the agreement is not perfect, as can be observed in Fig. 1. We shall come back to this small disagreement in the discussion of the errors in Section 3.3. Further evidence for the accuracy of DFT comes from the successful prediction of the b.c.c. to h.c.p. transition pressure [27,28], and comparison with the measured phonon density of states of the h.c.p. phase up to pressures of $\sim 150 \mathrm{GPa}$ [32]. With ab initio molecular dynamics, DFT calculations can also be performed on the liquid state, and we have reported extensive calculations both on pure liquid $\mathrm{Fe}[14,29,33]$ and on liquid $\mathrm{Fe} / \mathrm{S}$ and Fe/O alloys [34-36].

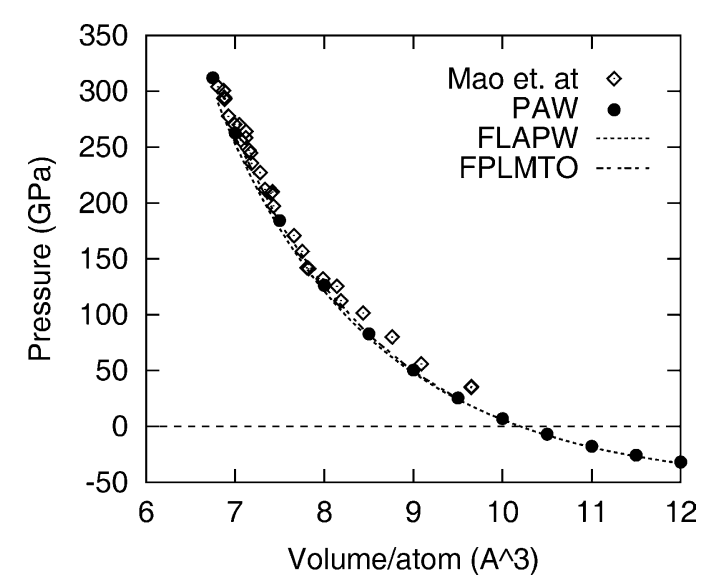

Fig. 1. Pressure as a function of atomic volume of h.c.p. Fe. Solid circles are present PAW calculations; dotted and chain curves are FLAPW and FPLMTO results of Ref. [27,28], respectively, diamonds are experimental values of Ref. [31]. 


\section{Free energies}

Having established the good quality description of a number of structural, magnetic and dynamical properties of iron we now move to the discussion of free energies.

At this point it is important to examine the issue of the accuracy needed in these calculations. As emphasised elsewhere [5,37], it is easy to show that in order to get the melting temperature with an error of less than $\approx 100 \mathrm{~K}$ the error on the difference of Gibbs free energies between solid and liquid has to be kept below $\approx 10 \mathrm{meV} /$ atom. This is a very small quantity, and it is much smaller than typical accuracy achievable with DFT techniques. However, we are not interested in absolute accuracies, but rather in the error in the difference of free energies between solid and liquid. In fact, DFT works very well for energy differences, especially in those cases where the structural and electronic properties of the different phases are very similar, like solid and liquid iron at high $p, T$ conditions. This means that we can rely on large cancellation of errors between the description of solid and liquid. It is conceivable therefore that, provided all other sources of technical errors are brought under control, the remaining error due to the actual implementation of DFT is small. We will come back to this point later in the article, where we will analyse more closely the possible DFT-GGA errors. Technical errors not related to DFT-GGA can in principle be made as small as wanted, and we have taken care that these were of the order of our target of $10 \mathrm{meV} /$ atom.

The techniques used to calculate free energies for solids and liquids have been reported and extensively discussed in previous papers $[5,6]$, so we only outline the main ideas here. The Helmholtz free energy of a system of $N$ atoms in a volume $V$ at temperature $T$ is given by

$$
\begin{aligned}
F= & -\kappa_{\mathrm{B}} T \ln \left\{\frac{1}{N ! \Lambda^{3 N}} \int \mathrm{d} \mathbf{R}_{1} \ldots \mathrm{d} \mathbf{R}_{N}\right. \\
& \left.\times \exp \left[-\beta U\left(\mathbf{R}_{1}, \ldots \mathbf{R}_{N} ; T\right)\right]\right\},
\end{aligned}
$$

where $\Lambda=h /\left(2 \pi M \kappa_{\mathrm{B}} T\right)^{1 / 2}$ is the thermal wavelength, with $M$ being the nuclear mass, $\beta=1 / \kappa_{\mathrm{B}} T, h$ is the Planck constant and $\kappa_{\mathrm{B}}$ the Boltzmann constant. We emphasise that $U\left(\mathbf{R}_{1}, \ldots \mathbf{R}_{N} ; T\right)$ is the free energy of the electrons in the system and therefore depends on $T$. A direct use of Eq. (1) to calculate the free energy of the system is impractical, as one would need to know the value of the (free) energy $U$ for every position of the atoms in the system. An alternative approach is to use the technique known as thermodynamic integration (see e.g. Ref. [38]), which is a completely general procedure for determining the difference of free energies $F_{1}-F_{0}$ of two systems whose total-energy functions are $U_{1}$ and $U_{0}$. The basic idea is that $F_{1}-F_{0}$ represents the reversible work done on continuously and isothermally switching the energy function from $U_{0}$ to $U_{1}$. To do this switching, a continuously variable energy function $U_{\lambda}$ is defined as

$U_{\lambda}=(1-\lambda) U_{0}+\lambda U_{1}$,

so that the energy goes from $U_{0}$ to $U_{1}$ as $\lambda$ goes from 0 to 1 . In classical statistical mechanics, the work done in an infinitesimal change $\mathrm{d} \lambda$ is

$\mathrm{d} F=\left\langle\mathrm{d} U_{\lambda} / \mathrm{d} \lambda\right\rangle_{\lambda} \mathrm{d} \lambda=\left\langle U_{1}-U_{0}\right\rangle_{\lambda} \mathrm{d} \lambda$,

where $\langle\cdot\rangle_{\lambda}$ represents the thermal average evaluated for the system governed by $U_{\lambda}$. It follows that

$F_{1}-F_{0}=\int_{0}^{1} \mathrm{~d} \lambda\left\langle U_{1}-U_{0}\right\rangle_{\lambda}$

In practice, this formula can be applied by calculating $\left\langle U_{1}-U_{0}\right\rangle_{\lambda}$ for a suitable set of $\lambda$ values and performing the integration numerically. The average $\left\langle U_{1}-U_{0}\right\rangle_{\lambda}$ is evaluated by sampling over configuration space. It is obvious that the final result for $F_{1}$ does not depend on the particular choice of the reference system, but the computational effort crucially depends on this choice. The reason for this is that in order to evaluate the quantity $\left\langle U_{1}-U_{0}\right\rangle_{\lambda}$ with a chosen statistical accuracy one needs to sample the phase space at a number of points which depends on the size of the fluctuations of $U_{1}-U_{0}$, so it is important to look for a reference system which minimises the size of these fluctuations.

\subsection{The liquid}

The problem of mimicking the fluctuations of ab initio energy $U_{1}$ in high-p/high- $T$ liquid Fe using a reference system was studied in detail in a previous paper [14]. We showed there that a $U_{0}$ consisting of a sum of pair potentials

$U_{0}=U_{\text {th }}+U_{\text {pair }}$,

in which

$U_{\text {pair }}=\frac{1}{2} \sum_{i \neq j} \phi\left(\left|\mathbf{R}_{i}-\mathbf{R}_{j}\right|\right)$,

can be arranged to mimic the fluctuations of $U_{1}$ very precisely, if we choose $\phi(r)$ to be a repulsive inverse-power potential $\phi(r)=B / r^{\alpha}$, with suitable values of $B$ and $\alpha$. In this expression for $U_{0}$, we have included a term $U_{\text {th }}$ which depends on thermodynamic state, but does not depend on the positions $\mathbf{R}_{i}$.

Calculations of $F$ were performed at 18 thermodynamic states spanning the conditions of $V$ and $T$ of the Earth's core. Convergence of the results with respect to size and $\mathbf{k}$-point sampling has been carefully checked with combined errors reduced to less than $\approx 5 \mathrm{meV} /$ atom; statistical errors in the evaluation of the integral in Eq. (2) were of the order of $\approx 2 \mathrm{meV} /$ atom. Free energies were then fitted to a polynomial function of $V$ and $T$, for the exact form of this function and more details we refer to our earlier paper [6]. The fitting 
reproduced the calculations to within a few milli-electron volt per atom at all points, so that we estimate the error on our free energies to be less than $10 \mathrm{meV} / \mathrm{atom}$.

\subsection{The solid}

The free energy of the solid is conveniently written as follows

$F_{\text {sol }}=F_{\text {perf }}+F_{\text {harm }}+F_{\text {anharm }}$,

where $F_{\text {perf }}$ is the free energy of the perfect non-vibrating crystal (it is a free energy because we take into account electronic excitations), $F_{\text {harm }}$ is the contribution due to the vibrations above zero temperature, which at core temperatures is very well approximated by the classical limit

$F_{\text {harm }}=-\left(\frac{3 \kappa_{\mathrm{B}} T}{\Omega_{\mathrm{BZ}} N_{i}}\right) \sum_{i} \int_{\mathrm{BZ}}\left(\ln \frac{\kappa_{\mathrm{B}} T}{\hbar \omega_{\mathbf{q}, i}}\right) \mathrm{d} \mathbf{q}$,

with errors of less than $1 \mathrm{meV} /$ atom at $T>3000 \mathrm{~K}$. Here $\Omega_{\mathrm{BZ}}$ is the volume of the BZ, $N_{i}$ is the number of atoms in the primitive cell and $\omega_{\mathbf{q}, i}$ is the frequency of the $i$ th phonon branch at wave vector q. The full quantum mechanics expression for the harmonic free energy is not more difficult to calculate than the classical limit. The reason for taking the classical limit is for consistency with the treatment of the liquid, for which the free energy has been calculated with molecular dynamics, using a classical treatment of the iron nuclei.

The calculation of $F_{\text {perf }}$ involves simple electronic structure calculations on perfect crystals; it can be easily done on small computers and it is virtually exact. The evaluation of $F_{\text {harm }}$ involves the calculations of the phonon frequencies $\omega_{\mathbf{q}, i}$ in the whole BZ, for which we used our own implementation [39] of the small displacement method $[5,40]$. In this method, a supercell is constructed and the atoms in the primitive cell are displaced by small amounts. The forces induced on all the atoms in the supercell are used to construct the force constant matrix, whose eigenvalues are related to the phonon frequencies. Errors on $F_{\text {harm }}$ arise from the finite size of the displacement of the atoms in the primitive cell, the finite size of the supercell and k-points sampling; we have taken care to reduce these errors below $3 \mathrm{meV} / \mathrm{atom}$.

The last term contributing to the total free energy of the solid, $F_{\text {anharm}}$, has been calculated using thermodynamic integration. We used as reference system a linear combination of the ab initio harmonic potential, $U_{\text {harm }}$ and the inverse power potential used as reference system for the liquid

$U_{\text {ref }}=a U_{\mathrm{IP}}+b U_{\mathrm{harm}}$,

with the two parameters $a$ and $b$ adjusted in order to minimise the fluctuations in the energy differences between the reference system and the full ab initio system. The anharmonic contribution to the free energy is relatively large at melting, it stabilises the solid by about $50 \mathrm{meV} / \mathrm{a}-$ tom, and contributes to raise the melting temperature by about $500 \mathrm{~K}$. Errors in the anharmonic contribution to the free energy are estimated to be about $10 \mathrm{meV} /$ atom, so that total errors in the free energy of the solid are of the order of $15 \mathrm{meV} /$ atom.

\subsection{The melting curve}

In this section we discuss the melting curve of $\mathrm{Fe}$ reported in Fig. 2 from 50 to $400 \mathrm{GPa}$. The solid black line is the result obtained by combining the free energies of solid and liquid, and we predict a temperature of $6350 \pm 300 \mathrm{~K}$ at $\mathrm{ICB}$, where the error quoted is the result of the combined statistical errors in the free energies of solid and liquid. This value is about $300 \mathrm{~K}$ lower than the values originally reported in Ref. [4], and the correction is the result of more precise calculations of the anharmonic contribution to the free energy of the solid, as reported earlier [5,6].

Also in Fig. 2, we show the ab initio melting curve reported by Laio et al. [7] and by Belonoshko et al. [8]. In addition, we compare with experimental melting curves or points obtained by shock experiments or by staticcompression using the DAC. DAC determinations of the melting curve of $\mathrm{Fe}$ and other transition metals have been performed by several research groups $[2,3,41,42]$. The early results of Williams et al. [1] lie considerably above those of other groups, and are now generally discounted. This still leaves a range of ca. $400 \mathrm{~K}$ in the experimental $T_{\mathrm{m}}$ at $100 \mathrm{GPa}$. Even allowing for this uncertainty, we acknowledge that our melting curve lies appreciably above the surviving DAC curves, with our $T_{\mathrm{m}}$ being above that of

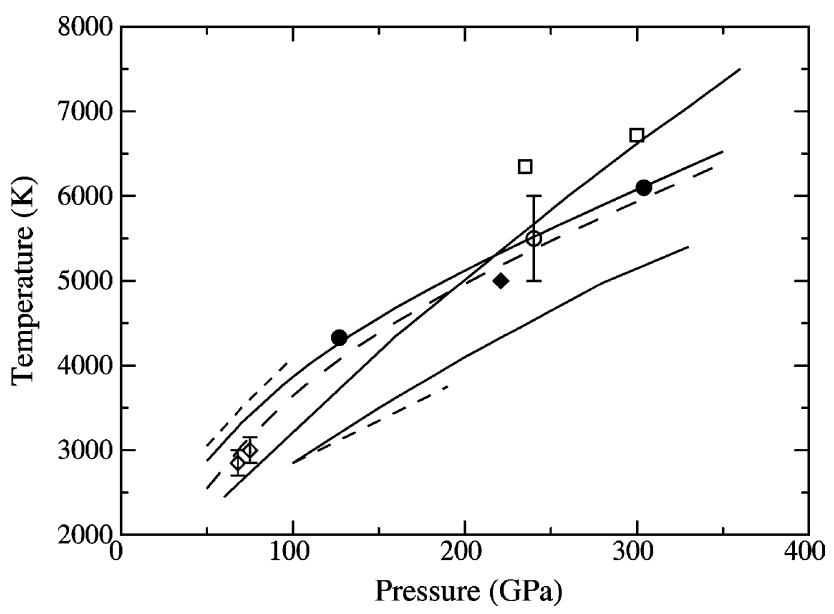

Fig. 2. Comparison of melting curve of Fe from present calculations with previous experimental and ab initio results: heavy solid and dashed curves: present work without and with free-energy correction (see text); black filled circles: present corrected coexistence results (see text); green curve: ab initio results of Ref. [7]; blue curve: ab initio results of Ref. [8]; grey and red dashed curves: DAC measurements of Refs. [1,2]; green diamonds: DAC measurements of Ref. [3]; black open squares, blue open circle and magenta diamond: shock experiments of Refs. [43-45]. Error bars are those quoted in original references. 
Shen et al. [3] by ca. $400 \mathrm{~K}$ at $100 \mathrm{GPa}$. We return to this discrepancy below.

Shock measurements should in principle be able to fix a point on the high-pressure melting curve at the thermodynamic state where melting first occurs on the Hugoniot. However, temperature is notoriously difficult to measure in shock experiments. The temperatures obtained by Yoo et al. [43] using pyrometric techniques are generally regarded as being too high by at least $1000 \mathrm{~K}$. This has been confirmed by our recent ab initio calculations [5] of Hugoniot temperature for h.c.p. Fe. We therefore disregard their data point on the melting curve. In the shock measurements of Brown and McQueen [44] and Nguyen and Holmes [45], no attempt was made to measure temperature, which was estimated using models for the specific heat and Grüneisen parameter; the approximate validity of these models is supported by our ab initio calculations [5] on h.c.p. Fe. However, the identification of the Hugoniot melting point has been hampered by the possible existence of a solidsolid transition. In their measurements of sound velocity on the Hugoniot, Brown and McQueen [44] believed that they had observed a solid-solid transition as well as a separate melting transition. We plot in Fig. 2 the point reported by Brown and McQueen [44] and the point obtained from the measurements of Nguyen and Holmes [45]. For the latter, the pressure of $221 \mathrm{GPa}$ is taken directly from their measurement of the onset of melting, while the temperature at this point is taken from our calculation of the Hugoniot temperature of the h.c.p. solid at this pressure, as reported in Ref. [5].

We want to discuss now possible systematic errors in our melting temperatures. First, we recall that even with the best available GGA for exchange-correlation energy the lowtemperature $p(V)$ relation for h.c.p. Fe is not in perfect agreement with experiment. This has been shown by a number of independent calculations using all-electron techniques [27,28] as well as pseudopotential [29] and PAW $[14,19]$ techniques, all of which agree closely with each other. Roughly speaking, the pressure is underpredicted by ca. $10 \mathrm{GPa}$ at near-ambient pressures and by ca. $8 \mathrm{GPa}$ in the region of $300 \mathrm{GPa}$. The pressure error can be thought of as arising from an error in the Helmholtz free energy, so that the true free energy $F_{\text {true }}$ can be written as $F_{\text {true }}=F_{\mathrm{GGA}}+\delta F$, where $F_{\mathrm{GGA}}$ is our calculated free energy and $\delta F$ is the correction. If we now neglect the temperature dependence of $\delta F$, and simply add $\delta F(V)$ to the calculated free energies of solid and liquid, this gives a way of gauging our likely errors. We find that this free-energy correction leads to a lowering of the melting curve by ca. $350 \mathrm{~K}$ in the region of $50 \mathrm{GPa}$ and by ca. $70 \mathrm{~K}$ in the region of $300 \mathrm{GPa}$.

The second error source we consider is the PAW implementation, and specifically our choice of the division into core and valence states, and the PAW core radii. At Earth's core pressures, the $3 p$ electrons, and to a lesser extent the $3 \mathrm{~s}$ electrons, must be treated as valence states.
But in our PAW implementation $[5,14]$ the $3 \mathrm{~s}$ and $3 \mathrm{p}$ are core states, with the associated error partially compensated for by inclusion of an effective pair potential. Moreover, the core radius of $1.16 \AA$ have used [5,14] may also affect the calculations, because under such high pressures and temperatures the atoms come so close that the cores overlap. We estimated this error in our earlier paper [6], and found that the effect is small, and stabilises the liquid by $6 \mathrm{meV} / \mathrm{atom}$, which has the effect of shifting the melting curve down by $\sim 60 \mathrm{~K}$.

As we show in Fig. 2, if we include both these corrections they bring our low-temperature melting curve into quite respectable agreement with the DAC measurements of Shen et al. [3], while leaving the agreement with the shock point of Nguyen and Holmes [45] essentially unaffected. There is still a considerable discrepancy with the DAC curve of Boehler [2].

\section{The coexistence method}

In this section we discuss the coexistence approach to the calculation of melting properties. With this method solid and liquid are simulated in coexistence, and the $p, T$ values extracted from the simulation give a point on the melting curve. The method can be implemented in a number of different ways. In the work of Morris et al. [46], coexisting solid and liquid $\mathrm{Al}$ were simulated with the total number of atoms $N$, volume $V$ and internal energy $E$ fixed. They showed that, provided $V$ and $E$ are appropriately chosen, the two phases coexist stably over long periods of time, and the average pressure $p$ and temperature $T$ in the system give a point on the melting curve. An alternative procedure would be to simulate at constant $(N, V, T)$. Yet another approach was used in the work of Laio et al. [7] on the high-pressure melting of $\mathrm{Fe}$; this used constant-stress simulations, with enthalpy almost exactly conserved. The approach of Belonoshko et al. [8] is different again. Here, the $(N, p, T)$ ensemble is used. The system initially contains coexisting solid and liquid, but since $p$ and $T$ generally do not lie on the melting line, the system ultimately becomes entirely solid or liquid. This approach does not directly yield points on the melting curve, but instead provides upper or lower bounds, so that a series of simulations is needed to locate the transition point. Whichever scheme is used, some way is needed of monitoring which phases are present. In the $(N, V, E)$ method of Morris et al. [46], graphical inspection of particle positions appears to have been used, supplemented by calculating of radial distribution functions to confirm the crystal structure of the solid. In the $(N, p, T)$ method of Belonoshko et al. [8], the primary diagnostic is the discontinuity of volume as the system transforms from solid to liquid.

Because the coexistence method is intrinsically computationally more demanding than the free energy approach, all the calculations of melting properties performed so far 
have been done using classical potentials, including the melting curves of $\mathrm{Fe}$ produced by Laio et al. [7] and Belonoshko et al. [8]. It follows that the melting properties are those of the potentials used in the calculations. In particular, the melting curves will in general be different from those that would be obtained by a direct coexistence simulation using first principles calculations. However, as we showed in our previous paper [6], it is possible to assess these errors and correct them. The difference in the melting temperature at a fixed pressure can be formulated in terms of the differences of the Gibbs free energies between the model potential and the ab initio system. At a chosen pressure $p$, the melting temperatures of the two systems are defined by: $G_{\mathrm{AI}}^{\mathrm{ls}}\left(p, T_{\mathrm{AI}}\right)=0$ and $G_{\text {mod }}^{\mathrm{ls}}\left(p, T_{\text {mod }}\right)=0$, where $G^{\mathrm{ls}}$ is the difference between the Gibbs free energies of liquid and solid. The ab initio and the model potential melting temperatures are different, in general, because $G_{\mathrm{AI}}^{\mathrm{ls}}\left(p, T_{\text {mod }}\right) \neq 0$. Working at the given pressure, we take the variable $p$ as read and express the ab initio value of $G^{\mathrm{ls}}$ as

$G_{\mathrm{AI}}^{\mathrm{ls}}(T)=G_{\mathrm{mod}}^{\mathrm{ls}}+\zeta \Delta G^{\mathrm{ls}}(T)$,

where with the symbol $\Delta$ we denote differences between the $\mathrm{ab}$ initio system and the model potential, and the parameter $\zeta$ is introduced so that the ab initio melting temperature $T_{\mathrm{AI}}$ can be written as a power series

$T_{\mathrm{AI}}=T_{\mathrm{mod}}+\zeta T^{\prime}+\ldots$.

Since the Gibbs free energies are equal in the two phases, this $T_{\mathrm{AI}}$ is the solution of $G_{\mathrm{AI}}^{\mathrm{ls}}(T)=0$, which is

$G_{\text {mod }}^{\mathrm{ls}}\left(T_{\text {mod }}+\zeta T^{\prime}+\ldots\right)+\zeta \Delta G^{\mathrm{ls}}\left(T_{\text {mod }}+\zeta T^{\prime}+\ldots\right)=0$.

Expanding in powers of $\zeta$ and equating powers, one obtains for $T^{\prime}$

$T^{\prime}=\Delta G^{\mathrm{ls}}\left(T_{\bmod }\right) / S_{\bmod }^{\mathrm{ls}}$

where $S_{\text {mod }}^{\mathrm{ls}}$ is the entropy of fusion of the model potential. Since entropies of fusion are on the order of $\kappa_{\mathrm{B}}$ per atom, then a difference $\Delta G^{\mathrm{ls}}$ of $10 \mathrm{meV} /$ atom implies a shift of melting temperature of ca. $100 \mathrm{~K}$, so that substantial errors will need to be corrected for unless the reference total energy function matches the ab initio one very precisely. The free energy differences $\Delta G^{\mathrm{ls}}$ can be calculated using thermodynamic integration, or if the model potentials mimic the ab initio system closely enough, a perturbational approach. If the calculations are performed in the $(N, V, T)$ ensemble it is easy to show that

$\Delta F=\langle\Delta U\rangle_{\bmod }-\frac{1}{2} \beta\left\langle\delta \Delta U^{2}\right\rangle_{\bmod }+\ldots$,

where $\delta \Delta U \equiv \Delta U-\langle\Delta U\rangle$, and the averages are taken in the model potential ensemble. The relation between $\Delta G$ and $\Delta F$, is readily shown to be

$\Delta G=\Delta F-\frac{1}{2} V \kappa_{T} \Delta p^{2}$, where $\kappa_{T}$ is the isothermal compressibility and $\Delta p$ is the change of pressure when $U_{\text {mod }}$ is replaced by $U_{\mathrm{AI}}$ at constant $V$ and $T$.

\subsection{The melting curve}

We now come to the results. The model potential for our coexistence simulations is the embedded-atom model (EAM) recently used by Belonoshko et al. [8] to calculate the high-pressure melting curve of Fe. Our coexistence simulations have originally been done in the $(N, V, E)$ ensemble with cells containing 8000 atoms, and we went up to 64,000 atoms to test size effects. More recently, we found that even cells containing 1000-2000 atoms are big enough to calculate melting temperatures with a size error of a few percentage at most. We performed the calculations at two different thermodynamic conditions, one with a volume per atom $\nu=7.12 \AA$, which is close to the average of the volumes of liquid and solid iron at ICB conditions, and a second one with a volume per atom $\nu=8.6 \AA$, which corresponds to a pressure of about $127 \mathrm{GPa}$, close to the pressure of the core mantle boundary [47]. We have carefully checked that the effect of small non-hydrostatic stresses does not affect significantly the calculated melting temperatures. In the top panel of Fig. 3 we report the temperature along the simulation for the system with $\nu=7.12 \AA$, and in the bottom panel the three diagonal components of the stress tensor. The three of diagonal components oscillate around zero average and we do not display them. From this simulation we extract the values $T=6550 \pm 100 \mathrm{~K}$ and $p=305 \pm 1 \mathrm{GPa}$, which are in

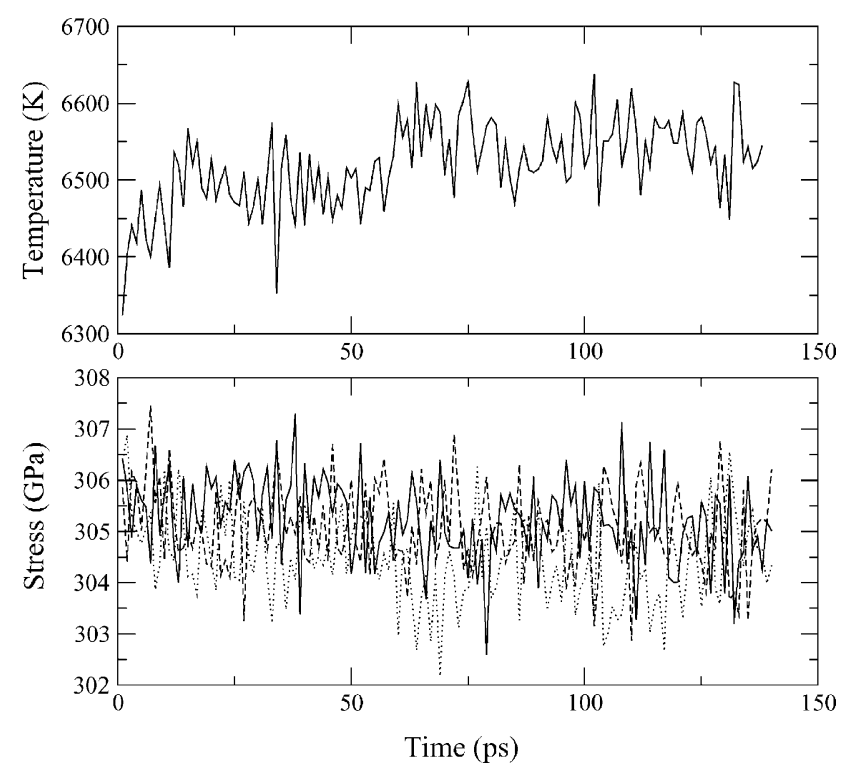

Fig. 3. Time variation of temperature (upper panel) and the three components of stress tensor $P_{x x}$ (solid curve), $P_{y y}$ (dashed curve) and $P_{z z}$ (dotted curve) (upper panel), during a simulation of solid and liquid $\mathrm{Fe}$ coexisting at a pressure of $305 \mathrm{GPa}$. Simulations were performed on a system of 8000 atoms using the embedded-atom potential of Belonoshko et al. [8], with $c / a$ ratio of the hexagonal cell equal to 1.66 . 
agreement with the value $6680 \mathrm{~K}$ reported by Belonoshko et al. [8] within the combined statistical errors. At $\nu=8.6$ $\AA$,we obtain $T=3650 \pm 50$ and $p=127 \pm 1$, which is also in agreement with the value $3720 \mathrm{~K}$ reported by Belonoshko et al. [8].

We now correct for the differences between this model potential and our ab initio system using Eqs. (14) and (15). Results for $\nu=7.12$ have been reported earlier in Ref. [9], where we found that free energy differences between the $a b$ initio and the EAM systems have the effect of stabilising the liquid with respect to the solid by about $35 \mathrm{meV} /$ atom compared with the EAM. Errors due to the finite size of the systems and k-points sampling have been carefully checked and reported in our previous paper [9]. Now, using the calculated value $S_{\text {mod }}^{\mathrm{ls}}=0.88 \kappa_{\mathrm{B}}$ /atom we obtain the first order correction $T^{\prime}=-450 \mathrm{~K}$, so that our corrected melting temperature at $p=305 \mathrm{GPa}$ is $6100 \pm 100 \mathrm{~K}$, in perfect agreement with the value $T=6100 \pm 300 \mathrm{~K}$ obtained with the free energy approach.

At $\nu=8.6$ we find opposite behaviour for the free energy differences, and in particular that the solid is stabilised with respect to the liquid by $50 \mathrm{meV} /$ atom. At these conditions we calculate $S^{\mathrm{ls}}=0.85 \kappa_{\mathrm{B}}$ /atom and we obtain a first order correction $T^{\prime}=680 \mathrm{~K}$, so that our corrected melting temperature at $p=127 \mathrm{GPa}$ is $4330 \pm 100 \mathrm{~K}$, which is also in excellent agreement with the value $4270 \pm 300 \mathrm{~K}$ obtained with the free energy approach.

These two points are also reported in Fig. 2.

\section{Discussion and conclusions}

Melting is defined by the locus of points where the Gibbs free energies of solid and liquid are equal. In this article, we have reported the melting curve of iron calculated using two different approaches. The first approach is based on the direct calculation of the Gibbs free energies of solid and liquid using ab initio techniques. In the second approach a model potential is fitted to ab initio calculations and melting is determined by solid-liquid coexistence simulations. In the latter approach the melting temperature obtained from the simulations is the one of the model potential, and here we have prescribed a way to correct for the possible differences with the ab initio melting temperature. We have shown that once these corrections are taken into account the results from the two methods agree closely.

We believe that this work settles the issue regarding the difference between our melting curve of iron $[4,6]$ and that calculated by Belonoshko et al. [8]. The other melting curve by Laio et al. [7] is also based on the coexistence method, and we argue that this also would come into close agreement with ours once the corrections described here and our previous paper [9] are applied.

Our melting curve agrees quite well with the shock datum of Brown and McQueen [44] and the point obtained from the measurements of Nguyen and Holmes [45]. It also agrees with the low-pressure DAC experiments of Shen et al. [3], but there is still a considerable discrepancy with the DAC data reported by Boehler [2].

\section{Acknowledgments}

Dario Alfè wishes to thank support from the Royal Society and from the Leverhulme Trust.

\section{References}

[1] Q. Williams, R. Jeanloz, J.D. Bass, B. Svendesen, T.J. Ahrens, Science 286 (1987) 181.

[2] R. Boehler, Nature 363 (1993) 534.

[3] G. Shen, H. Mao, R.J. Hemley, T.S. Duffy, M.L. Rivers, Geophys. Res. Lett. 25 (1998) 373.

[4] D. Alfè, M.J. Gillan, G.D. Price, Nature 401 (1999) 462.

[5] D. Alfè, G.D. Price, M.J. Gillan, Phys. Rev. B 64 (2001) 045123.

[6] D. Alfè, M.J. Gillan, G.D. Price, Phys. Rev. B 65 (2002) 165118.

[7] A. Laio, S. Bernard, G.L. Chiarotti, S. Scandolo, E. Tosatti, Science 287 (2000) 1027.

[8] A.B. Belonoshko, R. Ahuja, B. Johansson, Phys. Rev. Lett. 84 (2000) 3638.

[9] D. Alfè, M.J. Gillan, G.D. Price, J. Chem. Phys. 116 (2002) 6170.

[10] A.B. Belonoshko, R. Ahuja, B. Johansson, Nature 424 (2003) 1032.

[11] L. Vočadlo, D. Alfè, M.J. Gillan, I. Wood, J.P. Brodholt, G.D. Price, Nature 424 (2003) 536.

[12] D. Alfè, Phy. Rev. B 68 (2003) 064423.

[13] L. Vočadlo, D. Alfè, Phys. Rev. B 65 (2002) 214105.

[14] D. Alfè, G. Kresse, M.J. Gillan, Phys. Rev. B 61 (2000) 132.

[15] P. Hohenberg, W. Kohn, Phys. Rev. 136 (1964) B864. W. Kohn, L. Sham, Phys. Rev. 140 (1965) A1133. R.O. Jones, O. Gunnarsson, Rev. Mod. Phys. 61 (1989) 689. M.C. Payne, M.P. Teter, D.C. Allan, T.A. Arias, J.D. Joannopoulos, Rev. Mod. Phys. 64 (1992) 1045. M.J. Gillan, Contemp. Phys. 38 (1997) 115. R.G. Parr, W. Yang, DensityFunctional Theory of Atoms and MoleculesOxford University PressOxford (1989).

[16] Y. Wang, J. Perdew, Phys. Rev. B 44 (1991) 13298.

[17] J.P. Perdew, J.A. Chevary, S.H. Vosko, K.A. Jackson, M.R. Pederson, D.J. Singh, C. Fiolhais, Phys. Rev. B 46 (1992) 6671.

[18] P.E. Blöchl, Phys. Rev. B 50 (1994) 17953.

[19] G. Kresse, D. Joubert, Phys. Rev. B 59 (1999) 1758.

[20] S.H. Wei, H. Krakauer, Phys. Rev. Lett. 55 (1985) 1200.

[21] D. Vanderbilt, Phys. Rev. B 41 (1990) 7892.

[22] G. Kresse, J. Furthmüller, Phys. Rev. B 54 (1996) 11169

[23] G. Kresse, J. Furthmüller, Comput. Mater. Sci. 6 (1996) 15.

[24] D. Alfè, Comp. Phys. Commun. 118 (1999) 31.

[25] H.J. Monkhorst, J.D. Pack, Phys. Rev. B 13 (1976) 5188.

[26] C.S. Wang, B.M. Klein, H. Krakauer, Phys. Rev. Lett. 54 (1985) 1852.

[27] L. Stixrude, R.E. Cohen, D.J. Singh, Phys. Rev. B 50 (1994) 6442.

[28] P. Söderlind, J.A. Moriarty, J.M. Willis, Phys. Rev. B 53 (1996) 14063.

[29] L. Vočadlo, G.A. de Wijs, G. Kresse, M.J. Gillan, G.D. Price, Faraday Disc. 106 (1997) 205.

[30] L. Vočadlo, J. Brodholt, D. Alfè, M.J. Gillan, G.D. Price, Phys. Earth Planet. Inter. 117 (2000) 123.

[31] H.K. Mao, Y. Wu, L.C. Chen, J.F. Shu, A.P. Jephcoat, J. Geophys. Res. 95 (1990) 21737.

[32] H.K. Mao, J. Xu, V.V. Struzhkin, J. Shu, R.J. Hemley, W. Sturhahn, M.Y. Hu, E.E. Alp, L. Vočadlo, D. Alfè, G.D. Price, M.J. Gillan, M. Schwoerer-Böhning, D. Häusermann, P. Eng, G. Shen, H. Giefers, R. Lübbers, G. Wortmann, Science 292 (2001) 914. 
[33] G.A. de Wijs, G. Kresse, L. Vočadlo, D. Dobson, D. Alfè, M.J. Gillan, G.D. Price, Nature 392 (1998) 805.

[34] D. Alfè, M.J. Gillan, Phys. Rev. B 58 (1998) 8248.

[35] D. Alfè, M.J. Gillan, Phys. Rev. Lett. 81 (1998) 5161.

[36] D. Alfè, G.D. Price, M.J. Gillan, Phys. Earth Planet. Inter. 110 (1999) 191.

[37] G.A. de Wijs, G. Kresse, M.J. Gillan, Phys. Rev. B 57 (1998) 8223

[38] D. Frenkel, B. Smit, Understanding Molecular Simulation, Academic Press, San Diego, 1996

[39] D. Alfè, Program available at http://chianti.geol.ucl.ac.uk/ dario, 1998

[40] G. Kresse, J. Furthmüller, J. Hafner, Europhys. Lett. 32 (1995) 729.
[41] S.K. Saxena, G. Shen, P. Lazor, Science 264 (1994) 405.

[42] D. Errandonea, B. Schwager, R. Ditz, C. Gessmann, R. Boehler, M. Ross, Phys. Rev. B 63 (2001) 132104.

[43] C.S. Yoo, N.C. Holmes, M. Ross, D.J. Webb, C. Pike, Phys. Rev. Lett. 70 (1993) 3931

[44] J.M. Brown, R.G. McQueen, J. Geophys. Res. 91 (1986) 7485.

[45] J.H. Nguyen, N.C. Holmes, AIP Shock Compression Condens. Matter 505 (2000) 81.

[46] J.R. Morris, C.Z. Wang, K.M. Ho, C.T. Chan, Phys. Rev. B 49 (1994) 3109.

[47] A.M. Dziewonski, D.L. Anderson, Phys. Earth Planet. Inter. 25 (1981) 297. 